\title{
INFORME SOBRE LA COMISIÓN NACIONAL DE LA ESPECIALIDAD DE PSICOLOGÍA CLÍNICA
}

Después de su constitución en noviembre de 1999, la Comisión ha centrado su primer período de trabajo en la redacción de la Orden Ministerial que establece los procedimientos de aplicación de las disposiciones transitorias del Decreto de la Especialidad. La Orden entró en el período de tramitación en el mes de marzo, comprometiéndose el Ministerio a agilizar el procedimiento de forma que pudiera estar publicada en el Boletín Oficial en septiembre. En estos momentos se ha terminado el período de consultas a las Comunidades Autónomas, de forma que una vez tramitadas en la Secretaría General Técnica, se abrirá el periodo de audiencia pública que será de 15 días. Las alegaciones de las CCAA son, al parecer, meramente formales, por lo que no hay ningún problema para que el proceso siga adelante. A pesar de todo ello parece evidente la lentitud con la que se está gestionando todo el proceso.

Otros temas que se han trabajado y sobre los que se ha informado son: a) Recursos contra el Decreto de Psicología Clínica, b) Consejo Nacional de Especialidades Médicas, c) El libro del Residente, y d) Calendario de trabajo.

\section{A) Recursos contra el Decreto de Psicología Clínica}

Los recursos contra el Decreto de Psicología Clínica en los que la Asociación está personada, se encuentran en el siguiente punto:

- Asociación Gallega de psicólogos clínicos psicoterapeutas práctica privada: está pendiente de fallo, sin que se haya realizado periodo de pruebas periciales.

- Asociación Gallega de psicólogos clínicos privados: se ha contestado a la demanda.

- Consejo General de Colegios de Médicos: se ha contestado a la demanda y se ha abierto el periodo de pruebas periciales.

- Sociedades de Psiquiatría: hemos designado tres peritos (un catedrático de psicología, uno de psiquiatría y un profesional clínico) para informar sobre los siguientes puntos: 1) delimitación de los campos profesionales de los psiquiatras y los psicólogos, 2) elementos que se considera intervienen en el juicio clínicos diagnóstico de la enfermedad mental (biológicos, psicológicos y sociales). Hemos impugnado que el informe de la Real Academia de Medicina fuera admitido como prueba del Tribunal, como quería la parte demandante, y se ha admitido como prueba de parte, es decir, como prueba de las Sociedades de Psiquiatría.

\section{B) Consejo Nacional de Especialidades Médicas}

La Comisión solicitó, a raíz de su constitución, su inclusión en el Colegio Nacional de Especialidades Médicas, como el resto de las especialidades. La respuesta ha sido negativa con el razonamiento de que la Psicología Clínica no es una especialidad médica; igual respuesta ha recibido la Comisión Nacional de Radiofísicos. La opinión de la 
Comisión es que la respuesta parece, cuanto menos, incongruente, ya que deja a la Especialidad de Psicología Clínica en una situación totalmente ambigua, sin formar parte del organigrama institucional del Ministerios a pesar de ser una especialidad sanitaria oficialmente reconocida.

Ante esta situación, tanto en lo relativo a la lentitud de la tramitación de la Orden como a la negativa del Consejo Nacional de Especialidades Médicas, la Comisión valoró la situación como sumamente delicada y acordó dirigirse al Ministerio para solicitar la mayor prontitud en el proceso de tramitación, así como una clarificación de la situación institucional de la Comisión Nacional. Solicitó así mismo a las Asociaciones representadas en la misma que realizaran actuaciones en la misma línea frente al Ministerio.

\section{C) Libro del residente}

Se ha comenzado el plan piloto para poner a prueba, durante un cuatrimestre, el nuevo libro del residente en formación, común para Psicología Clínica y Psiquiatría, en cinco Unidades Docentes acreditadas, con el objetivo de introducir las modificaciones oportunas.

\section{D) Calendario de trabajo}

La Comisión se ha planteado un calendario de trabajo, para los próximos meses, en torno a varios temas:

- Revisión del programa de formación de la especialidad (PIR) y su relación con los planes de estudio de la licenciatura en Psicología.

- Áreas de Capacitación Específica. Se ha planteado trabajar en principio sobre tres áreas: psicoterapias, psicología clínica de niños y adolescentes y rehabilitación. El borrador sobre psicoterapias está ya bastante avanzado.

Madrid, 25 de octubre de 2000

Consuelo Escudero Representante AEPCP 